\title{
Modeling the Basic Cause-Effect Relationship between Supply Chain Events and Performance
}

\section{Conference Paper}

Author(s):

Heinecke, Georg; Köber, Jonathan; Kunz, Andreas (D); Lamparter, Steffen

Publication date:

2012

Permanent link:

https://doi.org/10.3929/ethz-a-007574272

Rights / license:

$\underline{\text { In Copyright - Non-Commercial Use Permitted }}$ 


\title{
Modeling the Basic Cause-Effect Relationship between Supply Chain Events and Performance
}

\author{
Georg Heinecke ${ }^{1,2}$, Jonathan Köber ${ }^{3}$, Andreas Kunz ${ }^{1}$, Steffen Lamparter ${ }^{2}$ \\ ${ }^{1}$ Institute of Machine Tools and Manufacturing, Swiss Federal Institute of Technology \\ Zurich, Switzerland \\ georghe@ethz.ch \\ kunz@iwf.mavt.ethz.ch \\ ${ }^{2}$ Siemens AG, Corporate Technology, Germany \\ georg.heinecke.ext@siemens.com \\ steffen.lamparter@siemens.com \\ ${ }^{3}$ CLAAS Selbstfahrende Erntemaschinen GmbH, Germany \\ jonathan.koeber@claas.com
}

\begin{abstract}
The increased integration of global supply networks with a reduction in stock and time buffers has raised their vulnerability to disturbances (i.e. events) that erode efficiency and competitiveness. The complex interrelationships in these networks lead to a cascade of knock-on effects that affect company performance in varying degrees. Hence, causality analysis is the key for early identification of critical events. To this end, a dynamic model is developed in this contribution. It describes the basic cause-effect relationship between events, their knock-on effects and company-internal performance indicators. To make the complexity of this task manageable, an effects-based classification of events is delineated from the EPCIS format. The utility and functionality of the model is illustrated on two examples. It is proposed that it serves as a basic outline for the logic foundation of the discovery component of supply chain event management (SCEM) systems.
\end{abstract}

\section{Introduction}

Over the past decades, lean management has brought a revolution to manufacturing that has reshaped supply chains and restructured factories (Holweg and Pil 2005). Loose bonds between companies have made way for strong partnerships of a few key-players with equal clout that conduct (joint) research and development of sophisticated modules. Ever more intertwined interdependencies of the material and information flows between these partners caused a shift from linear supply chains to complex, globalized supply networks (Wagner and Bode 2006). At the 
same time, factories have become capable of producing countless product variants with little time and stock buffers according to the build-to-order philosophy.

However, while lean management with prevalent just in time (JIT) deliveries requires smooth material flows, the reality of collapsed supply chains with idle productions illustrates that stochastic influences on a global scale not only cause uncertainty but eventually materialize in costly disruptions (Blackhurst et al 2005; Radjou et al 2002). Between 2000 and 2009, an annual average of 387 natural disasters were recorded worldwide (Guha-Sapir et al 2011). This equates to more than one disaster a day with potential adverse effects on supply networks. In 2010, Asia, the manufacturing center of the world, was struck by 134 disasters alone, reflecting a global share of $34.8 \%$ (Guha-Sapir et al 2011). Once labor disputes, demand volatility, supplier failures, transport delays, and others are added to this count, it comes as no surprise that today's complex supply networks are increasingly experiencing reliability problems. A company's performance and its customers are affected by unexpected disturbances that produce deviations of different magnitude between target and actual states (Otto 2003).

Supply chain event management (SCEM) addresses this problem. It is the practice of observing, prioritizing and reacting to events that occur during the operation of a supply chain (Tribowski et al 2009). Events in this context can be defined as disturbances that impede, in varying degrees, the execution of supply chain processes as they were originally scheduled. However, although SCEM addresses a fundamental problem, i.e. that intra- and inter-organizational processes are rarely executed as planned, it has received less attention from the academic community than supply chain risk management (SCRM) (Otto 2003). This is surprising given that supply glitches amplify along supply networks (Radjou et al 2002). Thus, if they are caught early when their effects are still insignificant, the worst effects upor downstream could be avoided.

Liu et al (2007) state, however, that this is the crux of the matter: Early identification of events with significant impacts on operational performance is difficult because its criticality to downstream processes depends on several dynamic factors. These factors change over time and continually alter the current state of the supply network and with that the criticality of an event. Thus, the same event at different points in time could have varying implications. For instance, the criticality of a two hour delivery delay depends as much on stock levels at the affected company as on the time buffer between scheduled delivery and start of production.

Liu et al (2007) pointed out that precisely these dynamics in integrated, complex supply chains may cause a storm of events and that "causality analysis is the key to controlling such a storm". In this contribution it is therefore argued - contrary to the common practice of defining static threshold values for event identification - that available event information (e.g. two hour delay) should be used to gauge the knock-on effects on a company's performance given the current state of the network. This evaluation can then be repeated in an online fashion when the event information is updated (e.g. three hour delay). To this end, a novel approach to event classification is presented and incorporated into the modeling of the cause-effect relationship between supply chain events, their knock-on effects, and 
company performance. It is proposed that the developed model serves as a basic outline for the logic foundation of event identifications through SCEM systems.

This contribution is structured as follows: Section 2 will introduce the SCEM concept and approaches for event identification. Due to the proximity of the research areas, it outlines similarities and differences between SCEM and SCRM. Section 3 then focuses on existing SCRM definitions and concepts and discusses their importance to the cause-effect relationship. Later an event classification approach is delineated. Section 4 outlines the cause-effect relationship model that is then evaluated in Section 5. Section 6 is devoted to some concluding remarks.

\section{Literature Review}

The origin of SCEM as a research area can be traced back to the beginning of the new millennium (Knickle 2001). Since then the concept has languished in the international academic community, rarely going beyond high-level, conceptual publications (e.g. Otto 2003). Meanwhile, in German-speaking countries, it has received more attention, albeit still in an insufficient depth (Straube et al 2007; Tribowski et al 2009). The lack of attractiveness is partly attributable to the popularity of a more mature, neighboring discipline: Supply chain risk management (SCRM). Although both essentially deal with events along the supply chain, however, they need to be clearly distinguished from each other. Roughly speaking, while SCRM aims at mitigating the potential of events (Tang 2006), SCEM focuses on mitigating the knock-on effects of critical events after they are observed (Bearzotti et al 2012; Otto 2003). To put it simply, SCEM comes into play when SCRM has failed. On the one hand SCEM is primarily concerned with the early identification of events and mitigation of their effects with short-term operational measures (Bearzotti et al 2012; Otto 2003). SCRM on the other hand deals with medium- and long-term strategic decisions that could mitigate the risk of e.g. a supplier failure by implementing a dual sourcing strategy (Tang 2006).

Unsurprisingly, given the state of the research area, SCEM specific mechanisms for early identification of critical events on the basis of the dynamic relationships between events and supply chain factors are largely missing. One exception is Liu et al (2007) where Petri nets were used to model event relationships in a supply chain to analyze the cause-effect relationships between consecutive events. It was shown that the variation of event parameters (e.g. probability of successful alternative sourcing) affects supply chain performance (fill rates, lead times, etc.). This led to the conclusion that by managing events it is possible to manage supply chain performance. However, investigations into the relationship between events and company performance were not part of the research.

Apart from cause-effect relationships, tools from statistical control can be used for event identification. It focuses on the differences between target and actual states. Since processes are stochastic, different realizations of certain (local) process parameters can be obtained. The resulting frequency curve allows for the defi- 
nition of threshold values below or above which a parameter is considered to be out of control and an alarm (to correct the process) is raised. This approach is of limited use in SCEM. First, an alarm suggests that effects have already considerably aggravated and containment options are limited or not existent anymore. Second, the analysis neglects dynamic supply chain factors like time and stocks. Breaching a threshold indicates a problem but it lacks information about the state of the following processes where e.g. enough security stock could be available.

Another impediment to event identification is a widespread lack of real-time status data (Günthner et al 2010). Supply chain transparency is a critical success factor and real-time monitoring systems are a prerequisite for effective SCEM systems. For various reasons these monitoring systems are rarely realized within companies and even less among partners (Curtin et al 2007; Günthner et al 2010). If they are present, reservations against data exchange are common, although the same companies trade large quantities of material (Lee and Whang 2000).

\section{Elements of the Cause-Effect Relationship}

Due to the dynamics of supply networks, it is argued that event identification based on threshold values of process parameters gives insufficient insight into the criticality of an event. Cause-effect relationships, however, facilitate decision making through the estimation of fluctuations in performance indicators, given a certain event and supply network state. The magnitude of change in these indicators, which depends heavily on dynamic factors (e.g. stocks, order situation, production schedule), enable decision makers to better evaluate the observed situation and, if necessary, devise counter-measures. Before these estimations are possible, crucial elements that constitute the cause-effect relationship are defined in the following subsections.

\subsection{Definition and Classification of Events and Their Basic Effects}

To enable an SCEM system to identify critical events from monitoring systems' data streams, the possible occurrences have to be defined first. This task can prove difficult for two main reasons. First, it is problematic to pinpoint the precise origin of an event, since frequently only the cascade of knock-on effects is noticed. Thus, the original source of an event may stay hidden. For instance, the bullwhip effect starts with small fluctuations in downstream demand and leads to much more severe fluctuations further upstream (Lee et al 1997). Second, it is unlikely that all possible interactions between the elements of a complex system can be foreseen, making it difficult to define all events that could affect it up front.

To facilitate the event definition process, risk classifications of SCRM that place specific occurrences into general classes can be transferred to SCEM. Cho- 
pra and Sodhi (2004) propose nine classes (disruptions, delays, systems, forecast, etc.). Jüttner (2005) identify three classes: Supply-side, demand-side and environmental risks. Gaonkar and Viswanadham (2004) propose an event classification based on the severity of their effects (deviations, disruptions, catastrophes). All classifications, however, leave it to the decision maker to specify concrete events. This approach seems arbitrary because in complex systems events can be overlooked and allocation is bound to be ambiguous at times.

To avoid these drawbacks and to reduce the complexity of the event definition process, it is proposed to employ a novel approach that is based on the immediate, object-related effects of an event on a company. They can be derived from the presence or absence of object-related observations at RFID-based monitoring points along the supply chain. We propose to describe the resulting primitive, realtime data streams of these observations with the EPCIS format (EPCIS 2007). It contains information that essentially answer the question of what object is when, where and why - the latter refers to the business context of the observation and is described by a business step ID. Hence, this information can be leveraged to notice cases when the supply chain in general and preceding logistics processes in particular fail to fulfill their purpose: To bring the right product, at the right time, to the right place, in the right quantity and the right quality (Figure 1). These five event classes can be easily identified by a comparison of reality (i.e. observation or its absence at a monitoring point) with plan information from a central database. For instance, suppose a traffic jam delays a truck delivery for two hours. The monitoring system first notices that the truck has not passed the monitoring point at the scheduled (i.e. planned) time. Once it passes the monitoring point, the delay can be quantified and expected effects on performance indicators gauged. Once the material arrives, however, the bulk-reading of the cargo, in conjunction with information from the database, reveals that too little was delivered. Thus, a delay becomes more severe when only part of the required material arrives.

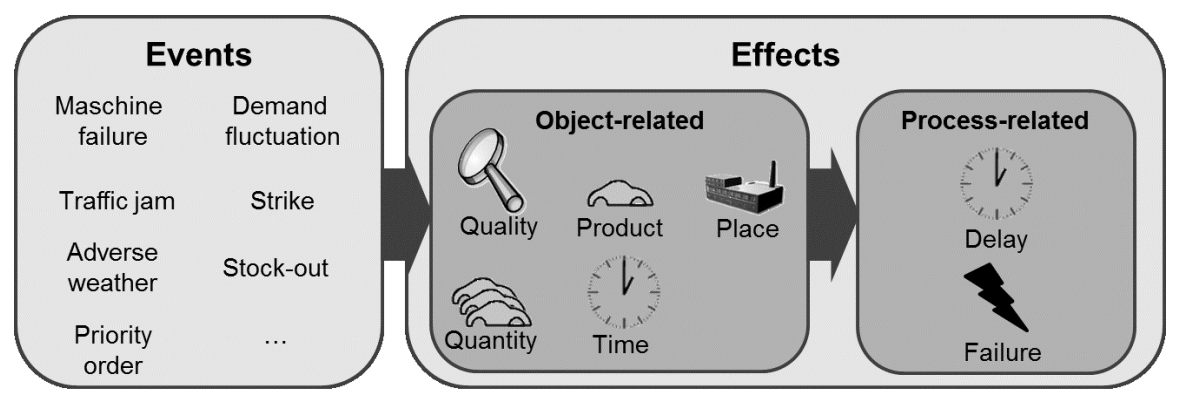

Fig. 1 Effects-based event classification

The example illustrates one advantage of using an event classification (Figure 1) that builds on the information provided through the EPCIS format - namely, once the resulting effects of each of these five classes on the processes of an affected company are examined more closely, it is apparent that they result in only 
two distinct cases of cause-effect relationships. First, material delivery delay constitutes an event where the observation at a specific point of the monitoring system was delayed. Second, material delivery failure constitutes an event where all or part of a shipment is useless (i.e. wrong product and/or quality) or unfulfilled (wrong quantity and/or place). These event classes also cover demand side risks such as priority orders or sudden demand fluctuations (e.g. wrong quantity delivered). For instance, a short-term increase in the order quantity by the customer results in a delivery failure when the supplier has insufficient material stocked for handling the increase, or the material is already on its way to the manufacturer.

It is apparent that an effects-based classification of events not only reduces (external) events to two distinct cases but, more importantly, relates them to time as the sole measuring unit. In short, the classification illustrates that all events, based on their effects, can be placed in a temporal context. Many of the external events that can occur at the supplier, however, can also happen at the company under consideration. These internal events (e.g. strike, machine failure, etc.) affect the shipment of the product and with this also the company's performance. Therefore, they have to be included when the cause-effect relationship of events on company performance is examined. Thus, two analog process-related event classes are proposed: material production delay and material production failure.

\subsection{Events and Vulnerability Drivers}

Wagner and Neshat (2010) state that "supply chain vulnerability is a function of certain supply chain characteristics and that the loss a firm incurs is a result of its supply chain vulnerability to a given supply chain disruption." Wagner and Bode (2006) identify five external drivers that increase vulnerability: single and global sourcing, customer and supplier dependence and supplier concentration.

Besides these external vulnerability drivers, internal ones also threaten a company's performance. For instance, small delivery delays quickly paralyze modern production systems due to their reliance on JIT deliveries that need little time and stock buffers. Furthermore, mass customization has led to an explosion of product variants, which has made the timely provisioning of specific material critical. At the same time, production systems lack flexibility that would allow a short-term adaption of the production program in case some material cannot be provided.

Although diverse, internal and external vulnerability drivers influence the event classes comparably by increasing the likelihood of occurrence and the severity of their effects. For instance, single sourcing of a specific part considerably raises the likelihood of a material delivery failure. Similarly, global sourcing of a part raises the likelihood of a material delivery delay due to larger distances. In contrast to SCRM, which aims at mitigating these drivers, SCEM incorporates their implications but otherwise regards them as fixed. Thus, an intelligent SCEM system preserves the advances of lean management (e.g. little time and stock buffers) while ensuring high performance in an event-prone environment. 


\subsection{Event Criticality and Performance Indicators}

To establish the cause-effect relationship, events have to be linked to meaningful indicators. Performance can be measured in various ways and literature proposes many different indicators (e.g. Gunasekaran et al 2001; Gunasekaran et al 2004). This contribution aims at relating an event and its knock-on effects to the success of a company. These effects are first noticed in production processes when material is delayed or missing. Therefore, rather than approaching performance measurement from a strategic, long-term perspective (e.g. customer satisfaction), the operational indicators of Nyhuis and Wiendahl (2006) are employed. These are affected first and allow a quick and concise estimation of the severity of an event.

Given a JIT delivery strategy with little buffers and no intervention, the most immediate effect of an event is a reduction of the work in process inventory as queues in front of production assets deplete. This reduction leads to i) shorter $c y$ cle times since waiting times are cut and ii) lower asset utilization due to increased idle times. Hence, also the production rate and overall output are cut. The customer notices the effects of an event when, through a lack of material, his outstanding orders cannot be fulfilled as scheduled and delivery delays accumulate.

\section{Modeling the Cause-Effect Relationship}

Systems dynamics is well-suited for the modeling and visualization of supply networks because the coherent notation can relate its many variables and their general interdependencies. According to Özbayrak et al (2007) "the ability of understanding the network as a whole, analyzing the interactions between the various components of the integrated system [...] make systems dynamics an ideal methodology for modeling supply chain networks."

The following subsections integrate the considerations from Section 3 into a simplified stock and flow diagram. First, the basic JIT manufacturing system with its interdependencies will be presented. It serves as baseline system. In the next subsection, the model will be enhanced through the integration of the event classes that affect a company. The final model helps in gauging the criticality of an event in a dynamic environment by quantifying their effects on performance indicators.

\subsection{Basic L Manufacturing System with Performance Indicators}

In very generic terms, a supply chain can be represented by several, consecutive accumulations (i.e. stocks) that are each increased by an inflow and decreased by an outflow (Sterman 2000). Operating according to the build to order principle, the supply chain of Figure 2 is initiated through product orders by the customer. 
These increase the backlog and equate to the material order rate of the manufacturer. This material order rate is the inflow for the stock of material that is on order but not yet delivered. The outflow is regulated by the delivery rate. It would normally increase the material inventory but is superfluous in a JIT system. Thus, the delivery rate directly equates to the production start rate that is responsible for the inflow into the work in process (WIP) inventory. It is drained by the production rate. The resulting product inventory is then drained by the ship rate. It equates to the fulfillment rate that decreases the backlog again. Thus, in a deterministic setting with constant flow rates the entire system quickly reaches equilibrium in which only a fixed time passes between the placement of the order by the customer and the fulfillment of the order by the manufacturer.

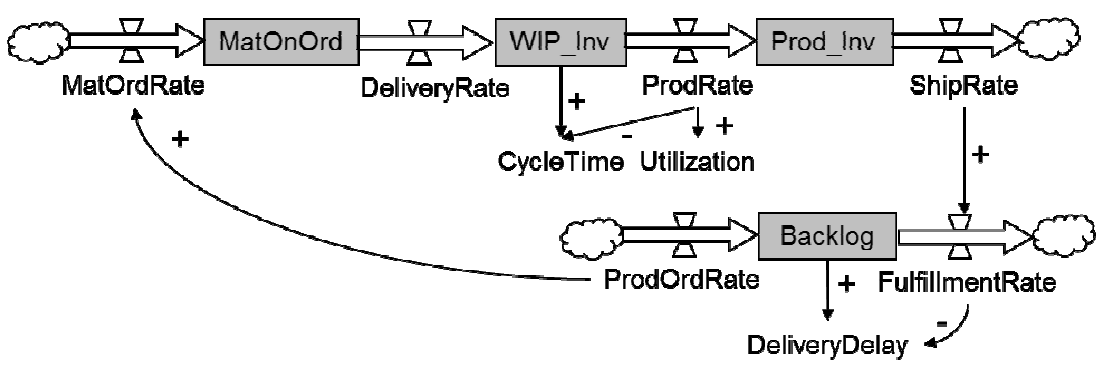

Fig. 2 JIT manufacturing system

The performance indicators of the system are stocks, cycle time, utilization, and delivery delays. Stocks are given by the WIP and production inventories. The cycle time is calculated using Little's Law, while utilization is derived from the production rate (Hopp and Spearman 2007). Lastly, the delivery delay is calculated from the backlog and the fulfillment rate.

\subsection{Event-prone JIT Manufacturing System}

Figure 3 incorporates the event classes and vulnerability drivers of Section 3 into the model. They directly influence the flow levels that are not constant anymore but fluctuate depending on the severity of the event. For instance, a tsunami will have a more profound but less sudden impact on the delivery rate than a traffic jam. The delivery rate is influenced, in the opposite direction, by the probabilities of a material delivery delay of different length and material delivery failure of different size: A larger probability leads to a lower average delivery rate as they occur more frequently over the simulation time. The production rate is similarly influenced by the internal event classes. All classes are affected by vulnerability drivers. For instance, global sourcing increases the distance between manufacturer and supplier, making a delivery delay or failure more likely and severe. 


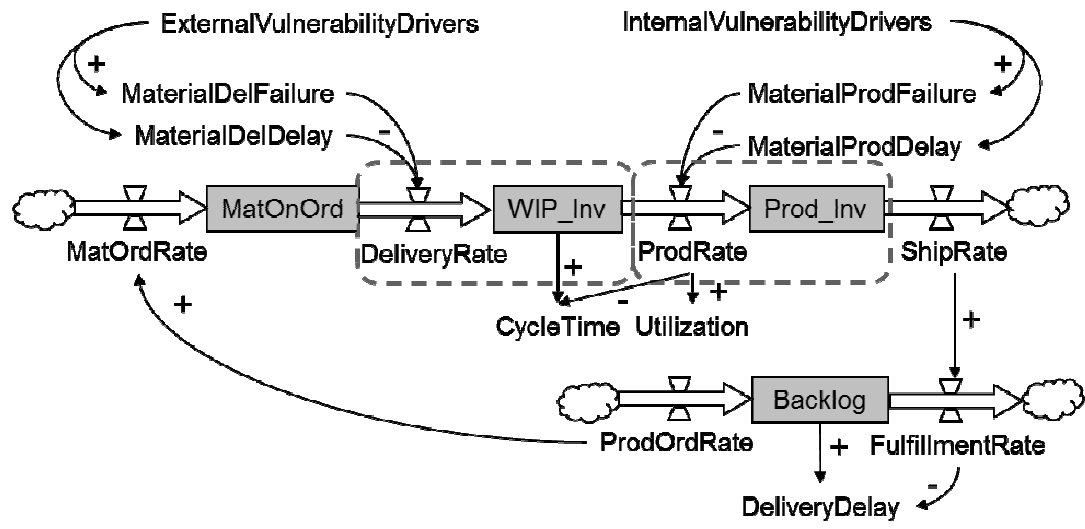

Fig. 3 Event-prone JIT manufacturing system

In Figure 3 the knock-on effects of events are now noticeable in a consecutive, time-dependent reduction first of the stocks and then of the flows of the system. Once the effects of an event have rippled through the manufacturing system, the shipping rate is reduced. Due to constant demand levels, several customer orders cannot be satisfied in this situation. The reduction in the fulfillment rate leads to an increase in the backlog. The manufacturing system is now clogged by orders that should already have been shipped. The orders that are supposed to be processed have to increasingly wait their turn, and delivery delays accumulate.

\section{Scenario Description and Performance Analysis}

To briefly verify and evaluate the usefulness of the presented concept, a supply "hiccup" (i.e. material delivery delay) of different length in a JIT environment is simulated. In the first scenario half of the required material for time $t+6$ is delayed and arrives at $t+7$. The second scenario increases this delay to two days. Thus, half the material is delayed in $t+6$ and $t+7$ and only arrives in $t+8$ and $t+9$ respectively. Both scenarios assume constant customer orders and production capacities of 10 units per day. The simulations were carried out using Vensim PLE.

Figure 4 illustrates the development of the WIP inventory. It is apparent that both systems reach equilibrium on day 3 where 10 units arrive and 10 units are processed. The one-(left diagram) and two-day (right diagram) delays then influence the system to the same effect, albeit in different magnitude: The average long-term WIP inventory increases. This circumstance is due to i) a lack of supplies inventory in JIT systems and ii) no flexibility in production capacity. The first results in a permanent loss of unused production capacity while the second prevents a new alignment between customer demand and WIP inventory. 

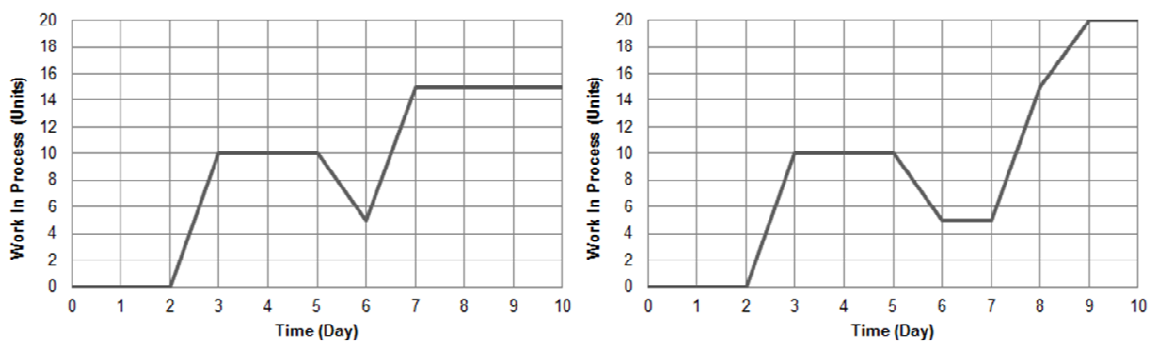

Fig. 4 Work in process (WIP) inventory of a one-(left) and two-day (right) delays

Figure 5 shows the development of the delivery delay. In both scenarios it also reaches equilibrium on day 3 when the average delay between order placement and order shipment is 3 days - i.e. the order to delivery time. In case of the oneday material delay (left) the delivery delay briefly doubles and then stays at 3.5 days. In the second scenario (right) it increases to 7 days before leveling out at 4 days. This analysis illustrates the fragility of a JIT system with no flexibility in production capacity. A brief delay permanently increases the WIP inventory and overall lead time as older orders are clogging the manufacturing system.
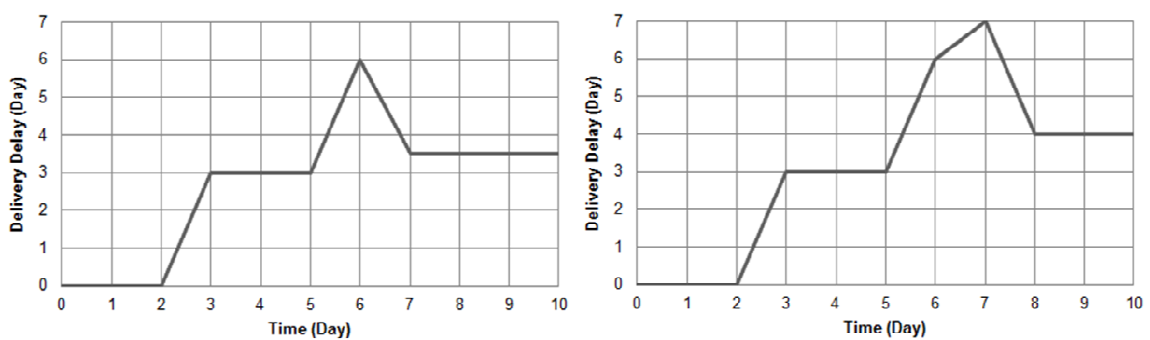

Fig. 5 Delivery delay of a one-(left) and two-day (right) delays

The examples illustrate that the basic logic of the model enables the isolation of the undistorted, performance-related effects of an event. The simulated performance analysis helps a decision maker, who faces the prospect of a one- or two-day material delivery delay, to make a more informed decision of whether the particular event is deemed critical to the operations and the success of the company. Obviously, this decision can be automated by defining thresholds for each indicator. If these are breached, an event is considered critical and planers can prepare counter-measures well before the effects of it reach the company. Besides costly emergency logistics schemes, measures then include, but are not limited to, economic alternatives such as a rescheduling of the order sequence by utilizing various flexibility potentials of the production. Hence, early event identification is critical because once the production sequence is fixed (i.e. frozen zone), rescheduling becomes an impossible task and affected products have to be reworked at high costs. 


\section{Concluding Remarks}

The increasing appearances of different supply chain events present a serious threat to the efficiency and competitiveness of global supply networks. This necessitates concepts and systems that can identify events early and react to their effects. To this end, a dynamic model is developed that describes the basic causeeffect relationship between events, their knock-on effects and performance indicators. To facilitate this task an effects-based classification of events on the basis of the EPCIS data format is proposed in this contribution. It is demonstrated that the model serves as a basic outline for the logic foundation of the event discovery component of supply chain event management (SCEM) systems. The simulation of two different delay events showed that the quantification of their performancerelated effects can help a decision maker to estimate their criticality in advance and more accurately. In future the proposed model is applied to an industrial case study to further investigate the validity of the proposed cause-effect relationship. Also, additional research will be done in regard to i) the combined effect an events when conflicting optimization goals are pursued, ii) the decision about the criticality of an event and iii) adequate response policies (e.g. rescheduling).

\section{References}

Bearzotti LA, Salomone E, Chiotti OJ (2012) An autonomous multi-agent approach to supply chain event management. International Journal of Production Economics 135(1):468-478

Blackhurst J, Craighead CW, Elkins D, Handfield RB (2005) An empirically derived agenda of critical research issues for managing supply-chain disruptions. International Journal of Production Research 43(19):4067-4081

Chopra S, Sodhi MS (2004) Managing risk to avoid supply-chain breakdown. MIT Sloan Management Review 46(1):53-62

Curtin J, Kauffman RJ, Riggins FJ (2007) Making the 'most' out of RFID technology: a research agenda for the study of the adoption, usage and impact of RFID. Information Technology and Management 8(2):87-110

EPCIS (2007) EPC Information Services Version 1.0.1 Specification

Gaonkar R, Viswanadham N (2004) A conceptual and analytical framework for the management of risk in supply chains. Proceedings - IEEE International Conference on Robotics and Automation pp 2699-2704

Günthner W, Meißner S, Conze M, Fischer R (2010) Stand und Entwicklung des RFID-Einsatzes in der Automobillogisitk: Ergebnisse einer empirischen Studie. RFID Anwenderzentrum München

Guha-Sapir D, Vos F, Below R, Ponserre S (2011) Annual Disaster Statistical Review 2010. Centre for Research on the Epidemiology of Disasters

Gunasekaran A, Patel C, Tirtiroglu E (2001) Performance measures and metrics in a supply chain environment. International Journal of Operations \& Production Management 21:71-87

Gunasekaran A, Patel C, McGaughey RE (2004) A framework for supply chain performance measurement. International Journal of Production Economics 87(3):333-347 
Holweg M, Pil FK (2005) The Second Century: Reconnecting Customer and Value Chain through Build-to-Order Moving Beyond Mass and Lean in the Auto Industry, MIT Press Books, vol 1. The MIT Press

Hopp W, Spearman M (2007) Factory Physics, 3rd edn. McGraw-Hill/Irwin

Jüttner U (2005) Supply chain risk management: Understanding the business requirements from a practitioner perspective. International Journal of Logistics Management 16(1):120-141

Knickle K (2001) Supply Chain Event Management - The next best thing to supply chain perfection. AMR Research Outlook

Lee HL, Whang S (2000) Information sharing in a supply chain. International Journal of Technology Management 20:373-387

Lee HL, Padmanabhan V, Whang S (1997) Information distortion in a supply chain: The bullwhip effect. Management Science 43(4):546-558

Liu R, Kumar A, van der Aalst W (2007) A formal modeling approach for supply chain event management. Decision Support Systems 43(3):761-778

Nyhuis P, Wiendahl HP (2006) Logistic production operating curves - Basic model of the theory of logistic operating curves. CIRP Annals - Manufacturing Technology 55(1):441-444

Otto A (2003) Supply chain event management: Three perspectives. The International Journal of Logistics Management 14(2):1-13

Radjou N, Orlov LM, Nakashima T (2002) Adapting to supply network change. Tech. rep., Forrester Research

Sterman JD (2000) Business Dynamics: Systems Thinking and Modeling for a Complex World, vol 53. Irwin McGraw-Hill

Straube F, Vogeler S, Bensel P (2007) RFID-based supply chain event management. In: RFID Eurasia, 2007 1st Annual, pp 1-5

Tang CS (2006) Perspectives in supply chain risk management. International Journal of Production Economics 103(2):451-488

Tribowski C, Goebel C, Guenther O (2009) EPCIS-based supply chain event management - a quantitative comparison of candidate system architectures. In: International Conference on Complex, Intelligent and Software Intensive Systems, pp 494-499

Wagner SM, Bode C (2006) An empirical investigation into supply chain vulnerability. Journal of Purchasing and Supply Management 12(6):301-312

Wagner SM, Neshat N (2010) Assessing the vulnerability of supply chains using graph theory. International Journal of Production Economics 126(1):121-129

Özbayrak M, Papadopoulou TC, Akgun M (2007) Systems dynamics modelling of a manufacturing supply chain system. Simulation Modelling Practice and Theory 15(10):1338-1355 